\title{
ESTRUTURA E RELAÇÕES AMBIENTAIS DE GRUPOS FLORÍSTICOS EM FRAGMENTO DE FLORESTA ESTACIONAL SUBTROPICAL ${ }^{1}$
}

\author{
Suzane Bevilacqua Marcuzzo², Maristela Machado Araújo³ e Solon Jonas Longhi ${ }^{4}$
}

\begin{abstract}
RESUMO - Este estudo teve por objetivos analisar a estrutura de grupos florísticos na vegetação arbóreaarbustiva de um trecho de encosta de Floresta Estacional Subtropical, no Parque Estadual Quarta Colônia, RS, e determinar fatores ambientais importantes na ocorrência das espécies, a fim de subsidiar programas de restauração de áreas degradadas como ecossistema de referência. Para isso, foi realizado estudo fitossociológico em 12 parcelas de $200 \mathrm{~m}^{2}(10 \times 20 \mathrm{~m})$, distantes $20 \mathrm{~m}$ entre si, distribuídas sistematicamente em quatro faixas de $100 \mathrm{~m}$ entre si, seguindo gradiente de topografia. Em cada parcela foram realizadas a identificação e a medição da circunferência à altura do peito (CAP) e da altura total dos indivíduos arbóreos e arbustivos, ou seja, com CAP $\geq 15 \mathrm{~cm}$, denominado componente arbóreo. Os dados do sub-bosque foram obtidos em parcelas circulares com raio de $1,78 \mathrm{~m}$, instaladas no centro das parcelas de 10 x $20 \mathrm{~m}$, medindo-se indivíduos com altura $\geq 30 \mathrm{~cm}$ e CAP $<15 \mathrm{~cm}$. As variáveis ambientais foram compostas pela declividade, pela intensidade luminosa, pelas características químicas e textura do solo. Os dados foram analisados segundo a estrutura e diversidade da vegetação por meio da análise de agrupamento pelo método TWINSPAN (Two-way Indicator Species Analysis) e da ordenação pelo método CCA (Análise de Correspondência Canônica). Foi identificada a formação de dois grupos florísticos. A composição de espécies de cada grupo pertence a diferentes estágios de sucessão natural, conforme histórico de uso agrícola, e, segundo suas características funcionais, podem ser indicadas para programas de restauração florestal.
\end{abstract}

Palavras-chave: Análise de agrupamento, Espécies indicadoras, Restauração florestal.

\section{STRUCTURE AND ENVIRONMENTAL RELATIONS OF FLORISTIC GROUPS IN TROPICAL DECIDUOUS FOREST FRAGMENT}

\begin{abstract}
The objective of this study was to determine floristic groups of the shrub-tree vegetation strata of an excerpt from Forest Subtropical Deciduous slope in Parque Estadual Quarta Colônia, Rio Grande do Sul, Brazil, to analyze their structure and to determine key environmental factors for plant species occurrence, in order to provide restoration programs of of degraded areas as reference ecosystem. A phytosociological survey was carried out for vegetation analysis on 12 plots of $200 \mathrm{~m}^{2}(10 \times 20 \mathrm{~m})$, systematically distributed on a topography gradient at $20 \mathrm{~m}$ interval, systematically distributed on four tracks of $100 \mathrm{~m}$ between them, following the gradient of topography. In each plot, it species was identified and it was measured circumference at breast height (CAP) and overall height of tree individuals, represented by those with CAP $\geq 115 \mathrm{~cm}$, named upper stratum. Understory strata information was obtained on circular plots of a radius of $1.78 \mathrm{~m}$, set up in the center of $10 \times 20 \mathrm{~m}$ plots, from individuals with height $\geq 30$ and CAP $<15 \mathrm{~cm}$. Environmental variables were steepness, light intensity and soil chemistry and texture. Data analyses of vegetation structure and diversity were evaluated by using TWINSPAN and environmental correlations of floristic composition by using CCA (Canonical Correspondence Analysis). It was identified two groups, with species composition belonging to different stages of natural succession, suggesting differences of agricultural land use history and soil chemical characteristics. For that, some species may be indicated in forest restoration programs.
\end{abstract}

Keywords: Cluster analysis, Indicator species, Forest restoration.

\footnotetext{
${ }^{1}$ Recebido em 23.07.2013 aceito para publicação em 17.01.2013.

${ }^{2}$ Departamento de Florestas e áreas protegidas, DEFAP, Brasil. E-mail:<smarcuzzo@ gmail.com>.

${ }^{3}$ Departamento de Ciências Florestais, Centro de Ciências Rurais, Universidade Federal de Santa Maria, RS, Brasil. E-mail:<araujo.maristela@gmail.com>e <longhi.solon@gmail.com>.
} 


\section{INTRODUÇÃO}

Dentro da região tropical e subtropical do planeta, pouco mais de quatro bilhões de hectares são cobertos por florestas, o que significa $31 \%$ da superfície terrestre total (FAO, 2010), e parte desse percentual é formada por Floresta Estacional. Atualmente, no Rio Grande do Sul as florestas sobre as encostas do planalto meridional encontram-se ameaçadas devido à conversão de alguns locais em áreas de produção rural e obras de utilidade pública, como usinas hidrelétricas.

Embora ainda existam remanescentes de Floresta Estacional Subtropical compondo as encostas do Planalto Meridional, poucas são as áreas protegidas na região, representadas por 2.422 ha apenas, os quais compõem a Reserva Biológica Estadual do Ibicuí Mirim e o Parque Estadual Quarta Colônia. Tal situação torna vulnerável a conservação da biodiversidade dessa tipologia florestal (SEMA, 2011).

A Floresta Estacional Subtropical é complexa em sua ecologia e apresenta diferentes relações entre grupos de espécies e hábitats e paisagem formada por mosaicos com acentuadas variações fitofisionômicas que acompanham gradientes ambientais, mesmo que ainda esteja em sua condição natural ou tenha pouca alteração antrópica. Silva e Scariot (2003) destacaram que o conhecimento da composição florística, estrutura e dinâmica da vegetação, associado às correlações com propriedades do solo, topografia e drenagem, permite que sejam feitas inferências sobre seu manejo, sobre as estratégias de conservação da biodiversidade e sobre a recuperação de áreas alteradas. Assim, a aplicação de técnicas de análise multivariada permite sintetizar, ordenar ou separar o conjunto de variáveis de forma otimizada, possibilitando melhor detecção de padrões estruturais em ambientes naturais (FELFILI et al., 2007).

Dessa forma, este estudo teve como objetivo geral analisar a vegetação arbórea-arbustiva, a regeneração e sua relação com as variáveis ambientais de solo em um trecho de encosta de Floresta Estacional Subtropical no Parque Estadual Quarta Colônia, a fim de subsidiar programas de restauração de áreas degradadas como ecossistema de referência. Para isso, foram identificados grupos florísticos na vegetação, analisando-se a influência de variáveis ambientais na distribuição das espécies e buscando espécies potenciais para restauração de áreas degradadas.

\section{MATERIAL E MÉTODOS}

\section{1. Área de estudo}

Este estudo foi realizado na área do Parque Estadual Quarta Colônia (PEQC), uma unidade de conservação de proteção integral com um território de $1.847,9$ ha, localizado nas coordenadas $29^{\circ} 37^{\prime} 40.80^{\prime \prime} \mathrm{Se}$ e $53^{\circ} 22^{\prime} 0.38^{\prime}$ 'O.

O Parque está situado nos Municípios de Agudo e Ibarama, no Rio Grande do Sul, na bacia hidrográfica do rio Jacuí. Caracteriza-se por apresentar trechos com floresta primária e trechos com vegetação secundária em estágio médio a avançado, oriundos do período anterior à desapropriação de pequenas propriedades. Os solos da região são predominantemente rasos, pouco desenvolvidos, sem horizonte $\mathrm{B}$ diagnóstico, diretamente assentados sobre a rocha e contêm material de rocha em decomposição (PEDRON; DALMOLIN, 2011). De acordo com o Sistema Brasileiro de Classificação de Solos, o solo da região é classificado como Neossolo Litólico (EMBRAPA, 2006).

O clima, segundo a classificação de Köppen, é subtropical, pertencente à variedade específica "Cfa", assim definida por apresentar temperatura média do mês mais frio entre $-3^{\circ}$ e $18^{\circ} \mathrm{C}$ (de junho a agosto) e, do mês mais quente, superior a $22^{\circ} \mathrm{C}$ (em janeiro) (NIMER, 1990).

A vegetação está inserida na região fitogeográfica de Floresta Estacional Subtropical (SCHUMACHER et al., 2011), representando um trecho de floresta de encosta do Parque, com cerca de 3 ha. O trecho, na face Oeste do morro, foi selecionado por representar o estágio médio a avançado de regeneração natural, servindo de comparativo da composição florística e estrutura em relação a áreas adjacentes em processo de restauração, após o plantio de espécies arbóreas nativas.

\subsection{Amostragem dos dados}

Para análise da vegetação, foi realizado um inventário das espécies arbóreas e arbustivas, em 12 parcelas de $200 \mathrm{~m}^{2}$ (10 x $20 \mathrm{~m}$ ), abrangendo $2.400 \mathrm{~m}^{2}$ (0,24 ha), distribuídas sistematicamente em gradiente de topografia e distanciadas $20 \mathrm{~m}$, em quatro faixas amostrais de 100 $m$ de distância entre si. Em cada parcela foram realizadas a identificação e a medição da circunferência à altura do peito (CAP) e da altura total dos indivíduos arbóreos e arbustivos (indivíduos com CAP $\geq 15 \mathrm{~cm}$ ), denominado componente arbóreo. Os indivíduos do sub-bosque (altura 
$\geq 30 \mathrm{cmeCAP}<15 \mathrm{~cm}$ ) foram medidos em parcelas circulares com raio de 1,78 m (FINGER, 1992), instaladas no centro das 12 parcelas de $10 \times 20 \mathrm{~m}$, totalizando $120 \mathrm{~m}^{2}$.

No caso de plantas não identificadas em campo, coletou-se material botânico que foi conduzido ao herbário da UFSM para averiguação por especialistas. As espécies foram identificadas de acordo com o sistema de classificação Angiosperm Phylogeny Group III (APG III, 2009).

As variáveis ambientais observadas foram obtidas por meio da análise de solos, da declividade e da intensidade luminosa do local. Para isso, foram coletadas cinco subamostras de solo, na profundidade de 0-20 $\mathrm{cm}$ em todas as parcelas, enviadas ao Laboratório de Análise de Solos do Departamento de Solos da UFSM e analisadas de acordo com a metodologia proposta por Tedesco et al. (1995). Assim, os dados determinados foram: argila, $\mathrm{pH}$ do solo ( $\mathrm{pH}$ em água), acidez potencial (H+Al), fósforo pelo método Mehlich (PMehlich), potássio extraível (K), matéria orgânica (MOS), cálcio (Ca), magnésio (Mg) e alumínio trocável (Al), capacidade de troca de cátions (CTC) e relações molares $\mathrm{Ca} / \mathrm{Mg}$, $(\mathrm{Ca}+\mathrm{Mg}) / \mathrm{K}$ e $\mathrm{K} /(\mathrm{Ca}+\mathrm{Mg})^{1 / 2}$.

A declividade do solo foi medida com hipsômetro de Blume Leiss, através da leitura em graus nas extremidades da porção central de cada parcela, obtendo-se a declividade média.

\subsection{Análise dos dados}

A abrangência florística em relação ao esforço da amostragem foi avaliada pela curva de acumulação de espécies obtida por aleatorização da entrada das parcelas, através do programa PC-ORD versão 5.0. Os resultados indicam que a amostragem foi suficiente para representar a vegetação (Figura 1).

As espécies encontradas foram enquadradas de acordo com os grupos ecológicos, conforme revisão bibliográfica (BACKES; IRGANG, 2002; CARVALHO, 2003, 2006; GRINGS; BRACK, 2009), e, de forma complementar, foram utilizados a classificação de Budowski (1965), com que foram reconhecidos quatro grupos (pioneira, secundária inicial, secundária tardia e clímax), e outros parâmetros de crescimento, tolerância à sombra e dispersão de sementes.

A diversidade florística foi estimada pelo índice de diversidade de Shannon (H') e a equabilidade, pelo coeficiente de Pielou (J) (BROWER; ZAR, 1984).
A formação de grupos entre as parcelas foi analisada por meio do Two-way Indicator Species Analysis (TWISPAN), que estabelece agrupamentos entre parcelas e espécies correlacionadas, verificando padrões na distribuição de espécies associados indiretamente às condições locais da área (HILL, 1979). A matriz de vegetação foi construída a partir das espécies que apresentaram mais de três indivíduos amostrados, somando 32 espécies nas 12 parcelas para o componente arbóreo (CA) e 19 espécies em 12 parcelas circulares para o sub-bosque (SB). Foram excluídas as espécies raras, pois, segundo Causton (1998), indivíduos menos abundantes contribuem muito pouco ou nada para a ordenação. Os níveis de corte para caracterizar as pseudoespécies foram 1 ( 1 ou 2 indivíduos), 2 (3 a 4), 3 (5 a 6), 4 (7 a 8), 5 (9 a 10) e 6 (mais de 11 indivíduos), determinados com base nas densidades das espécies em cada amostra. Assim, determinada espécie pode ser representada por seis pseudoespécies, com diferente número de indivíduos, como a pseudoespécie 1 da Actinostemum concolor, que apresenta um ou dois indivíduos em determinada parcela; já a pseudoespécie 2 tem de três a quatro e, assim, sucessivamente até a pseudoespécie 6, que apresenta mais de 11 indivíduos.

A partir da classificação, os grupos foram caracterizados em relação à estrutura horizontal com base nos parâmetros fitossociológicos (densidade, dominância, frequência e valor de importância), conforme descrito em Felfili e Resende (2003).

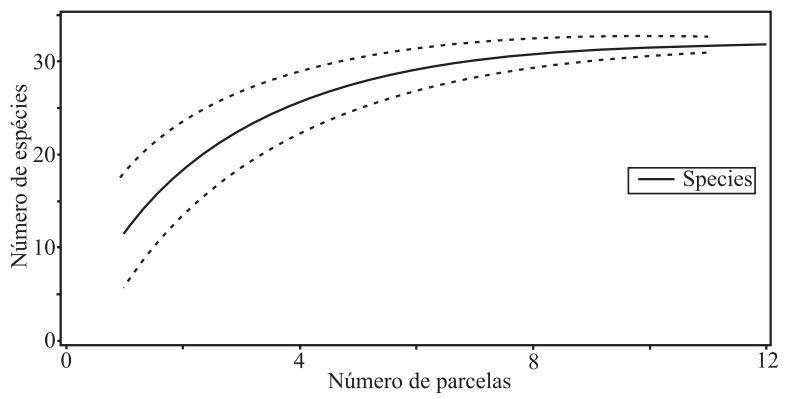

Figura 1- Curva de espécies x área (linha contínua) com intervalos de confiança de 95\% (linhas tracejadas), no trecho de floresta estacional decidual do Parque Estadual Quarta Colônia (área amostral composta de 12 parcelas de $200 \mathrm{~m}^{2}$, totalizando $0,24 \mathrm{ha}$ ).

Figure 1-Curve species x area (continuous line) with confidence intervals of $95 \%$ (dashed lines), in the stretch of the deciduous forest Parque Estadual Quarta Colônia ( sample area composed of 12 plots of 200 square meters, with a total of $0.24 \mathrm{ha}$ ).

Revista Árvore, Viçosa-MG, v.37, n.2, p.275-287, 2013

Revista Arvore, Viçosa-MG, v.37, n.2, p.275-287, 2013

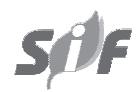


Para analisar as correlações ambientais dos gradientes com a vegetação arbórea e arbustiva, foi utilizada a análise de correspondência canônica (CCA) (FELFILI et al., 2011). A matriz de espécies foi a mesma utilizada para o TWINSPAN, entretanto os valores de abundância foram transformados pela raiz quadrada, visando atenuar o efeito dos desvios provocados por abundância elevada (PALMER,1993). A matriz ambiental consistiu, inicialmente, das seguintes variáveis: argila, $\mathrm{pH}$ em água, acidez potencial $(\mathrm{H}+\mathrm{Al}), \mathrm{P}$ extraível, $\mathrm{K}$ trocável, matéria orgânica (MO\%), Ca, $\mathrm{Mg}$ e Al trocáveis, $\mathrm{CTC}$, relações molares $\mathrm{Ca} / \mathrm{Mg},(\mathrm{Ca}+\mathrm{Mg}) / \mathrm{Ke} \mathrm{K} /(\mathrm{Ca}+\mathrm{Mg})^{1 /}$ ${ }^{2}$ e declividade.

Após análise preliminar, foram removidas variáveis fracamente correlacionadas, com valor de correlação inferior a 0,4, totalizando-se para análise do componente arbóreo nove variáveis. Dessas, oito de solo (Ca, Mg, $\mathrm{H}+\mathrm{Al}$, CTC efetiva, \% MO, \% argila, PMehlich e K) e uma de relevo (declividade). Para o sub-bosque, restaram nove variáveis que se referem ao solo (Ca, $\mathrm{Mg}, \mathrm{CTC}$ efetiva, \% MO, \% argila, PMehlich, $\mathrm{K}$, relações molares $(\mathrm{Ca}+\mathrm{Mg}) / \mathrm{K}$ e $\mathrm{K} /(\mathrm{Ca}+\mathrm{Mg})^{1 / 2}$. A significância das correlações entre as matrizes foi analisada pelo teste de permutação de Monte Carlo, através do programa PC-ORD for Windows versão 5 (MCCUNE; MEFFORD, 2006). Após as análises, a parcela 10 foi excluída por se tratar de um afloramento rochoso sem vegetação.

\section{RESULTADOS}

Foram observados 1.608 indivíduos.ha ${ }^{-1}$ (ind.ha $^{-1}$ ) no componente arbóreo, distribuídos em 21 famílias, 40 gêneros e 48 espécies. No sub-bosque foram estimados 1.770,83 ind.ha ${ }^{-1}$, distribuídos em 22 famílias, 35 gêneros e 42 espécies. Juntos, o componente arbóreo e o subbosque apresentaram 56 espécies. Dessas, 44 espécies são comuns aos dois estratos, cinco ocorrem apenas no sub-bosque e sete estão presentes apenas no componente arbóreo. Adicionalmente, a identificação das sinúsias da vegetação e a classificação das espécies nos grupos ecológicos definidos por Budowski resultaram em 10 espécies pioneiras, 24 secundárias iniciais e 22 secundárias tardias.

O índice de diversidade de Shannon (H') para o componente arbóreo apresentou valor de 3,0 e o índice de equabilidade de Pielou (J') igual a 0,78. No subbosque, o índice de diversidade de Shannon foi H'= 2,60 e o índice de equabilidade de Pielou foi J'=0,69.
A partir da classificação da vegetação do componente arbóreo (CA), foram observados dois grupos distintos para o trecho de floresta estudado. A primeira divisão, com autovalor de $\mathbf{0 , 4 6 7 4}$, gerou o grupo $\mathbf{1}_{\mathbf{C A}}$ (parcelas $6,7,8,9,11$ e 12) que se distribui sobre local de abundante material rochoso fragmentado e declivoso; e o grupo $\mathbf{2}_{\mathrm{CA}}$ (parcelas 1, 2, 3, 4, 5 e 13), localizado em área de maior luminosidade, devido à existência de clareiras no local.

O grupo $\mathbf{1}_{\mathrm{CA}}$ apresentou a espécie Trichilia claussenii C.DC. como espécie indicadora, também representada pelas pseudoespécies $1,2,3$ e 4, o que evidencia grande abundância na área.

Como espécies preferenciais, destacam-se Annona rugulosa (Schltdl.) H. Rainer, Balfourodendron riedelianum (Engl.) Engl., Phytolacca dioica L., Sebastiania brasiliensis Spreng., Seguieria aculeata L. e Trichilia elegans A. Juss., representadas pela pseudoespécie 1, o que indica baixa densidade por parcela. O mesmo vale para Allophylus edulis (A.St.Hil., Cambess. \& A. Juss.), Actinostemon concolor Spreng. e Trichilia clausseni, representadas pelas pseudoespécies 2, 3 e 4. Já a espécie Actinostemon concolor é representada pela pseudoespécie 6 , pois em três parcelas ocorreram mais de 11 indivíduos.

Ainda referente ao grupo $\mathbf{1}_{\mathbf{C A}}$ a hierarquia das espécies que melhor descreve a estrutura da floresta é: Actinostemon concolor, Trichilia claussenii e Allophylus edulis, representando o valor de importância (VI\%) de 30,2. Contudo, essa contribuição se deve à sua ampla distribuição na área, como é o caso da Trichilia clausseni $(\mathrm{FA}=100)$, e ao elevado número de indivíduos, como é o caso da Actinostemon concolor (Tabela 1). Apenas Allophylus edulis apresentou maiores valores de dominância (DoA).

O grupo $2_{\text {CA }}$ apresentou Matayba elaeagnoides Radlk. como indicadora e como preferencial pelas pseudoespécies 1, 2, 3. Entre outras preferenciais, estão as espécies Cupania vernalis Cambess., representada pelas pseudoespécies 1, 2, 3, 4, e Banara tomentosa Clos., Cedrela fissilis Vell., Citrus sp., Dalbergia frutescens (Vell.) Britton, Lonchocarpus nitidus (Vogel) Benth., Machaerium paraguariensis Hassl., Myrsine umbellata Mart., Nectandra lanceolata Nees. e Strychnos brasiliensis (Spreng) Mart., representadas pela pseudoespécie 1, Campomanesia guazumifolia (Cambess.) O. Berg. e Ocotea puberula (Rich.) Nees., representadas pelas pseudoespécies 1 e 2; e Casearia 
sylvestris Sw. e Cordia americana (L.) Gottshling \& J.E.Mill., ambas representadas pelas pseudoespécies 2 e 3.

Na estrutura desse grupo, puderam-se observar os maiores valores de importância nas espécies Nectandra megapotamica (Spreng.) Mez. (10,89), Nectandra lanceolata $(9,45)$, Cupania vernalis $(9,35)$, Ocotea puberula $(7,73)$ e Casearia sylvestris $(6,04)$ (Tabela 1$)$. O gênero Nectandra predominou, pois havia Nectandra lanceolata em $6,155 \mathrm{~m}^{2} \cdot \mathrm{ha}^{-1}$ e Nectandra megapotamica em 5,224 $\mathrm{m}^{2} \cdot \mathrm{ha}^{-1}$, indicando elevada área basal em ambas as espécies. Comparativamente às demais, Nectandra megapotamica apresentou frequência de $100 \%$, ou seja, esteve presente em todas as parcelas (FA).

Quando foi classificada a vegetação do sub-bosque (SB), também foi observada a formação de dois grupos, cuja maioria das parcelas coincide com aquelas que formaram os grupos do componente arbóreo (CA). Contudo, observou-se que no grupo $\mathbf{1}_{\mathbf{S B}}$ ficaram classificadas (autovalor de $\mathbf{0 , 4 2 4 7}$ ) as parcelas 5, $6,8,11,12$ e, no grupo $2_{\mathbf{s B}}$ as parcelas $1,2,3,4$, $7,9,13$, ou seja, essas apresentam semelhança florística e estrutural entre si e diferentes entre grupos. No grupo $\mathbf{1}_{\mathbf{S B}}$, a espécie Actinostemon concolor se apresenta como espécie indicadora e, como preferenciais, as espécies Seguieria aculeata L. (representada pela pseudoespécie 1) e Actinostemon concolor (representada pelas pseudoespécies 2, 3, 4 e 5), o que reflete a presença expressiva desse grupo no sub-bosque. Tal resultado é corroborado pelos maiores parâmetros de densidade e frequência de Actinostemon concolor, seguida pela de Trichilia elegans.

O grupo $2_{\mathrm{SB}}$ apresentou como espécie indicadora Cupania vernalis e como espécies preferenciais Allophylus edulis, Allophylus guaraniticos (A. St.Hil.) Radlk., Cabralea canjerana (Vell.) Mart., Campomanesia guazumifolia, Casearia sylvestris, Machaerium paraguariensis, Myrsine umbellata Mart. e Parapiptadenia rigida (representadas pela pseudoespécie 1) e Cupania vernalis e Trichilia claussenii (representadas pela pseudoespécie 2). $\mathrm{Na}$ estrutura da floresta, Cupania vernalis apresentou a melhor hierarquização devido aos seus elevados valores de densidade (DA) e frequência (FA).

Ainda por meio da Análise de Correspondência Canônica CCA, foi possível verificar a influência de variáveis ambientais sobre a formação de grupos distintos na área de estudo.

Tabela 1 - Estrutura horizontal e grupo sucessional das espécies arbóreo-arbustivas encontradas em trecho de Floresta Estacional Subtropical no Parque Estadual Quarta Colônia, RS.

Table 1 - Horizontal structure and successional group of shrub-tree species found in Subtropical Deciduous Forest in Parque Estadual Quarta Colônia, RS, Brazil.

\begin{tabular}{|c|c|c|c|c|c|c|c|c|c|c|c|c|c|}
\hline \multirow{3}{*}{ Espécies } & \multirow{3}{*}{ GS } & \multicolumn{6}{|c|}{ GRUPO 1} & \multicolumn{6}{|c|}{ GRUPO 2} \\
\hline & & \multicolumn{4}{|c|}{ Componente arbóreo } & \multicolumn{2}{|c|}{ Sub-bosque } & \multicolumn{4}{|c|}{ Componente arbóreo } & \multicolumn{2}{|c|}{ Sub-bosque } \\
\hline & & DA & FA & DoA & $\mathrm{VI} \%$ & DA & FA & $\mathrm{DA}$ & FA & DoA & VI\% & DA & FA \\
\hline Actinostemon concolor & $\mathrm{St}^{3}$ & 391,67 & 83,33 & 1,693 & 12,5 & 10083 & 41,6 & 25 & 50 & 0,04 & 1,60 & 1917 & 41,66 \\
\hline Allophylus edulis & $\mathrm{Si}^{2}$ & 150 & 66,66 & 2,831 & 8,42 & & & 91,6 & 50 & 0,78 & 3,56 & 916,7 & 41,66 \\
\hline Casearia sylvestris & $\mathrm{Si}^{1}$ & 50 & 50 & 0,709 & 3,295 & & & 183 & 83,3 & 0,92 & 6,04 & 333,3 & 16,66 \\
\hline Cordia am & $\mathrm{Si}^{1}$ & 25 & 50 & 2,635 & 5,10 & 166,6 & 16,6 & 158 & 83,3 & 1,26 & 5,99 & 333,3 & 25 \\
\hline Cupania vernalis & $\mathrm{Si}^{3}$ & 75 & 50 & 1,067 & 4,25 & 583,3 & 25 & 266 & 100 & 2,28 & 9,35 & 4917 & 58,33 \\
\hline Hybanthus bigibbosus & $\mathrm{St}^{3}$ & & & & & 333,3 & 16,6 & & & & & 1583 & 41,66 \\
\hline Nectandra lanceolata & $\mathrm{St}^{3}$ & & & & & & & 66,6 & 66,6 & 6,15 & 9,45 & & \\
\hline Nectandra megapotamica & $\mathrm{Si}^{3}$ & 83,33 & 66,66 & 2,681 & 6,84 & 166,6 & 8,33 & 166 & 100 & 5,22 & 10,8 & 250 & 16,66 \\
\hline Ocotea puberula & $\mathrm{Pi}^{3}$ & 8,33 & 16,66 & 0,574 & 1,33 & 166,6 & 8,33 & 91,6 & 83,3 & 3,87 & 7,73 & 83,33 & 8,333 \\
\hline Seguieria aculeata & $\mathrm{Si}^{3}$ & 33,33 & 50 & 0,168 & 2,29 & 583,3 & 25 & 8,33 & 16,6 & 0,01 & 3,42 & 166,7 & 8,333 \\
\hline Trichilia claussenii & $\mathrm{St}^{3}$ & 216,67 & 100 & 1,553 & 9,19 & & & 8,33 & 16,6 & 0,01 & 0,53 & 1833 & 25 \\
\hline Trichilia elegans & $\mathrm{St}^{3}$ & 25 & 33,33 & 0,061 & 1,52 & 666,6 & 25 & 8,33 & 16,6 & 0,01 & 0,53 & 1500 & 50 \\
\hline Total (12 espécies) & & 1058 & & 13,97 & 54,8 & 12750 & & 1075 & & 20,6 & 59,1 & 1383 & \\
\hline Total (44 espécies) & & 525 & & 13,36 & 44,3 & 2000 & & 825 & & 8,72 & 39,0 & 6833 & \\
\hline Total & & 1583 & & 27,33 & 99,1 & 14751 & & 1900 & & 29,3 & 98,1 & 2066 & \\
\hline
\end{tabular}

Onde: $\mathrm{G} . \mathrm{S}=$ grupo sucessional: $\mathrm{Pi}=$ pioneira, $\mathrm{Si}=$ secundária inicial; $\mathrm{St}=$ secundária tardia. ${ }^{1} \mathrm{Backes}$ e Irgang $(2002) ;{ }^{2} \mathrm{Carvalho}(2003)$; ${ }^{3}$ Grings e Brack (2009); DA= densidade absoluta; FA= frequência absoluta; DoA= dominância absoluta; VI\%= valor de importância. 
A análise de distribuição das parcelas e espécies com relação às variáveis ambientais, para o componente arbóreo, indicou autovalores de 0,383 e 0,255 nos dois primeiros eixos de ordenação, respectivamente. $\mathrm{O}$ eixo 1 explicou 20,2\% da variância, enquanto o eixo 2 correspondeu a $13,4 \%$. Esses resultados indicam que as variáveis ambientais utilizadas explicam 33,6\% da variância na distribuição das espécies; os outros $66,4 \%$ dizem respeito a fatores não mensurados e que ocorrem aleatoriamente na natureza.

No sub-bosque, as variáveis ambientais responderam de forma mais expressiva, pois os dois primeiros eixos de ordenação apresentaram os seguintes autovalores: 0,557 (eixo 1) e 0,320 (eixo 2). O eixo 2 explicou $49,7 \%$ da variância, e o eixo 3 correspondeu a $14,5 \%$, verificando-se variância acumulada de $64,2 \%$. Adicionalmente, o teste de permutação de Monte Carlo indicou alta correlação entre a abundância de espécies e as variáveis ambientais para o componente arbóreo e o sub-bosque nos eixos de ordenação $(p=0,010)$. As variáveis mais correlacionadas com o primeiro eixo de ordenação foram aquelas relacionadas à química e física do solo (Tabela 2).

A propósito, a área de estudo apresenta altos teores de elementos trocáveis como Ca $\left(27 \mathrm{cmol}_{\mathrm{c}} / \mathrm{dm}^{3}\right), \mathrm{Mg}$ $\left(5,4 \mathrm{cmol} / \mathrm{dm}^{3}\right), \mathrm{K}\left(320 \mathrm{mg} / \mathrm{dm}^{3}\right)$ e saturação de bases $(\mathrm{V}>50 \%)$, teores de CTC $\left(31,6 \mathrm{cmol} / \mathrm{dm}^{3}\right)$ na faixa de pH 6,0-6,5.
A ordenação das unidades amostrais e das espécies do componente arbóreo no primeiro e no terceiro eixo sugere a influência das características químicas do solo (Figura 2), assim como no sub-bosque nos eixos 2 e 3 (Figura 3).

\section{DISCUSSÃO}

De fato, a riqueza florística do trecho em estudo apresentou semelhança com outras áreas das encostas do rebordo meridional da Serra Geral, na região central do Estado do Rio Grande do Sul. Entretanto, demonstra apresentar uma riqueza inferior quando comparada com a Floresta do Alto Uruguai.

Scipioni et al. (2009) encontraram, na Depressão Central do RS, 59 espécies na regeneração natural em fragmento de floresta na Reserva Biológica do Ibicuí Mirim em 0,28 ha, e 60 espécies no componente arbóreo amostrado em 1,12 ha. Já Giehl e Jarenkow (2008) observaram, em uma área de 1,0 ha, 82 espécies no Parque Estadual do Turvo, que está situado sobre a Floresta Estacional do Alto Uruguai.

Da mesma forma, o índice de diversidade de Shannon (H') apresentou valor comparável a outros índices encontrados em estudos realizados nas florestas estacionais do Estado do Rio Grande do Sul. Os resultados refletem alta diversidade para a Floresta Estacional Subtropical, o que pode estar relacionado ao histórico da área, uma vez que há trechos de floresta que,

Tabela 2 - Coeficientes de correlação entre as variáveis ambientais e os eixos de ordenação encontrados para a regeneração e o componente arbóreo de trecho de Floresta Estacional Decidual no Parque Estadual Quarta Colônia, RS.

Table 2 - Correlation coefficients between the environmental variables and the ordination axes found for regeneration and the upper stratum of Deciduous Forest in a stretch in Parque Estadual Quarta Colônia, RS, Brazil.

\begin{tabular}{|c|c|c|c|c|c|c|}
\hline \multirow{3}{*}{ Variáveis ambientais } & \multicolumn{3}{|c|}{ Componente arbóreo } & \multicolumn{3}{|c|}{ Sub-bosque } \\
\hline & \multicolumn{6}{|c|}{ Correlação } \\
\hline & Eixo 1 & Eixo 2 & Eixo 3 & Eixo 1 & Eixo 2 & Eixo 3 \\
\hline Declividade (Dec) & 0,258 & $-0,248$ & 0,112 & $-0,278$ & $-0,111$ & 0,192 \\
\hline Cálcio (Ca) & $-0,511$ & 0,351 & 0,062 & $-0,062$ & 0,194 & 0,656 \\
\hline Magnésio (Mg) & $-0,511$ & 0,343 & 0,292 & $-0,017$ & 0,282 & 0,619 \\
\hline Acidez potencial (HAl) & $-0,563$ & $-0,079$ & $-0,006$ & $-0,007$ & $-0,188$ & 0,311 \\
\hline CTC efetiva & $-0,503$ & 0,352 & 0,106 & $-0,049$ & 0,199 & 0,661 \\
\hline Matéria orgânica (MO) & $-0,373$ & 0,244 & 0,061 & $-0,035$ & $-0,015$ & 0,591 \\
\hline Argila & $-0,556$ & $-0,055$ & 0,194 & $-0,105$ & 0,376 & 0,651 \\
\hline Fósforo (PMehlic) & $-0,412$ & 0,017 & $-0,476$ & $-0,123$ & 0,166 & $-0,005$ \\
\hline Potássio (K) & 0,244 & 0,300 & 0,064 & 0,102 & $-0,530$ & 0,430 \\
\hline Relações molares $(\mathrm{Ca} / \mathrm{Mg} / \mathrm{K})$ & 0,055 & 0,153 & 0,002 & $-0,159$ & 0,757 & 0,173 \\
\hline Relações molares (K/Ca/Mg) & 0,123 & 0,233 & 0,034 & 0,147 & $-0,722$ & 0,152 \\
\hline
\end{tabular}

Revista Árvore, Viçosa-MG, v.37, n.2, p.275-287, 2013 
provavelmente, não tenham sido explorados pela agricultura devido à intensa declividade da encosta. Na região central do Estado, na Reserva Biológica do Ibicuí Mirim, Scipioni et al. (2011) encontraram o valor de H'=3,21 e J'=0,78. Já Kilca e Longhi (2011) encontraram H'=3,08 em florestas secundárias do Rebordo do Planalto Meridional.

No sub-bosque, o índice de diversidade de Shannon e o índice de equabilidade de Pielou apresentaram valores menores quando comparados aos encontrados por Scipioni et al. (2009), que encontraram $H^{\prime}=3,21$ e J'=0,78 na Reserva Biológica do Ibicuí Mirim. Connel e Lowman (1989) explicaram que, em florestas caracterizadas pela monodominância, as baixas diversidades, em torno de $\mathrm{H}^{\prime}=2,3$ a 2,5 , podem ser atribuídas à alta plasticidade fenotípica das espécies que as compõem, ou seja, à ampla distribuição no ambiente. Entretanto, estudos realizados por Narvaes et al. (2005),

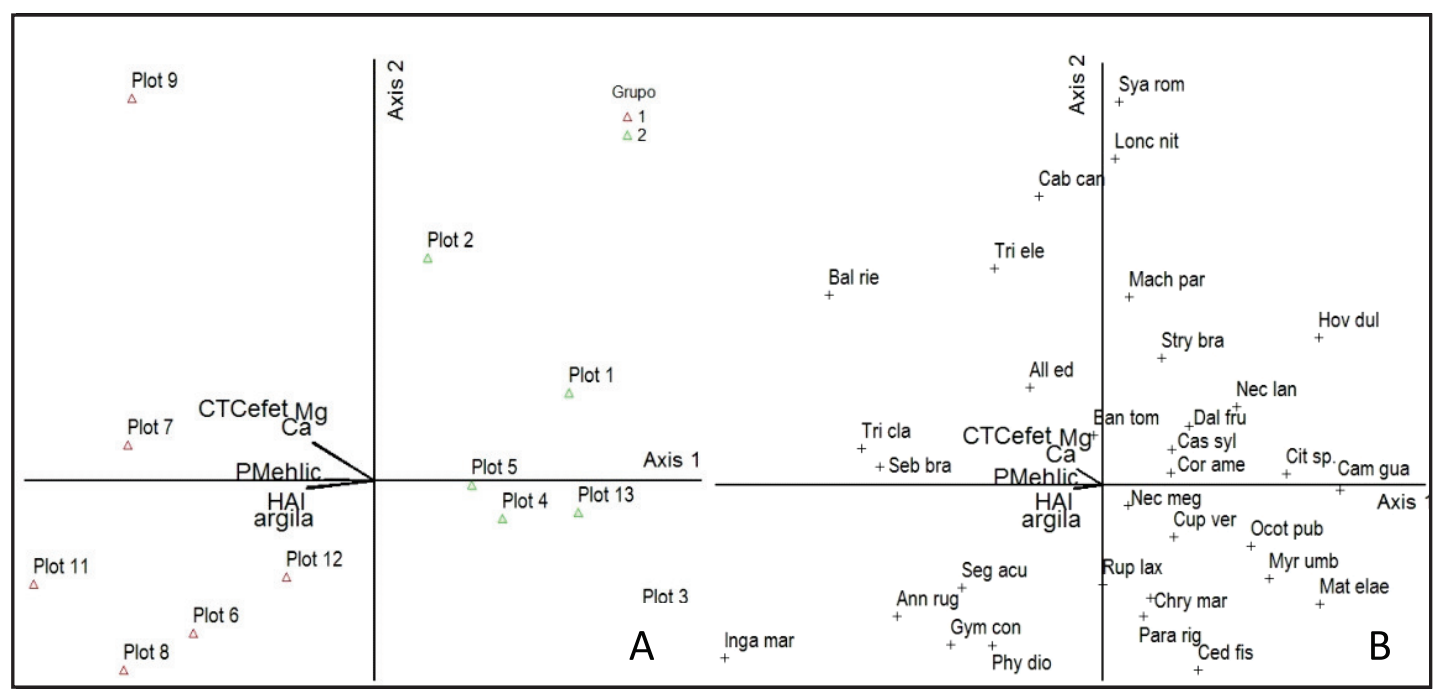

Figura 2 - Distribuição das parcelas (A) e espécies (B) de acordo com os eixos 1 e 2 da CCA, gerada a partir dos dados de abundância das espécies ocorrentes no componente arbóreo e das variáveis ambientais em um trecho de Floresta Estacional Subtropical no Parque Estadual Quarta Colônia, no RS. Axis:eixos; Plot:parcelas; CTCefet: capacidade de troca catiônica efetiva; $\mathrm{Ca}$ : cálcio; PMehlic: fósforo; $\mathrm{HAl}$ :acidez potencial; $\mathrm{Mg}$ : magnésio; Bal rie:Balfourodendron riedelianum; Tri ele:Trichilia elegans; Cab can:Cabralea canjerana; All ed:Allophylus edulis; Tri cla:Trichilia clausseni; Seb bra:Sebastiania brasiliensis; Lonc nit:Lonchocarpus nitidus; Mach par:Machaerium paraguaiensis; Ban tom:Banara tomentosa; Stry bra:Strychnos brasiliensis; Hov dul:Hovenia dulcis; Nec lan: Nectandra lanceolata; Dal fru:Dalbergia frutescens; Cas syl:Casearia sylvestris; Cor ame:Cordia americana; Cit sp.:Citrus sp; Cam gua:Campomanesia guazumifolia; Nec meg:Nectandra megapotamica; Cup ver:Cupania vernalis; Ocot pub:Ocotea puberula; Myr umb:Myrsine umbellata; Mat elae:Matayba elaegnoides; Ced fis:Cedrela fissilis; Chry mar:Chrysophyllum marginatum; Para rig:Parapiptadenia rigida; Rup lax:Ruprechtia laxiflora; Seg acu:Seguieria aculeata; Ann rug:Annona rugulosa; Gym con:Actinostemon concolor; Inga mar:Inga marginata; Phy dio:Phytolacca dioica.

Figure 2 - Plots distribution and species according to axis 1 and 2 of the CCA, generated from the data of abundance of species found in the upper strata and environmental variables in a Semideciduous Forest in Parque Estadual Quarta Colônia, RS. CTCefet: effective cátion Exchange capacity; Ca: calcium; PMehlic: phosphorus; HAl:potential acidity; Mg: magnesium; Bal rie:Balfourodendron riedelianum; Tri ele:Trichilia elegans; Cab can:Cabralea canjerana; All ed:Allophylus edulis; Tri cla:Trichilia clausseni; Seb bra:Sebastiania brasiliensis; Lonc nit:Lonchocarpus nitidus; Mach par:Machaerium paraguaiensis; Ban tom:Banara tomentosa; Stry bra:Strychnos brasiliensis; Hov dul:Hovenia dulcis; Nec lan: Nectandra lanceolata; Dal fru:Dalbergia frutescens; Cas syl:Casearia sylvestris; Cor ame:Cordia americana; Cit sp.:Citrus sp; Cam gua:Campomanesia guazumifolia; Nec meg:Nectandra megapotamica; Cup ver:Cupania vernalis; Ocot pub:Ocotea puberula; Myr umb:Myrsine umbellata; Mat elae:Matayba elaegnoides; Ced fis:Cedrela fissilis; Chry mar:Chrysophyllum marginatum; Para rig:Parapiptadenia rigida; Rup lax:Ruprechtia laxiflora; Seg acu:Seguieria aculeata; Ann rug: Annona rugulosa; Gym con:Actinostemon concolor; Inga mar:Inga marginata; and Phy dio:Phytolacca dioica. 


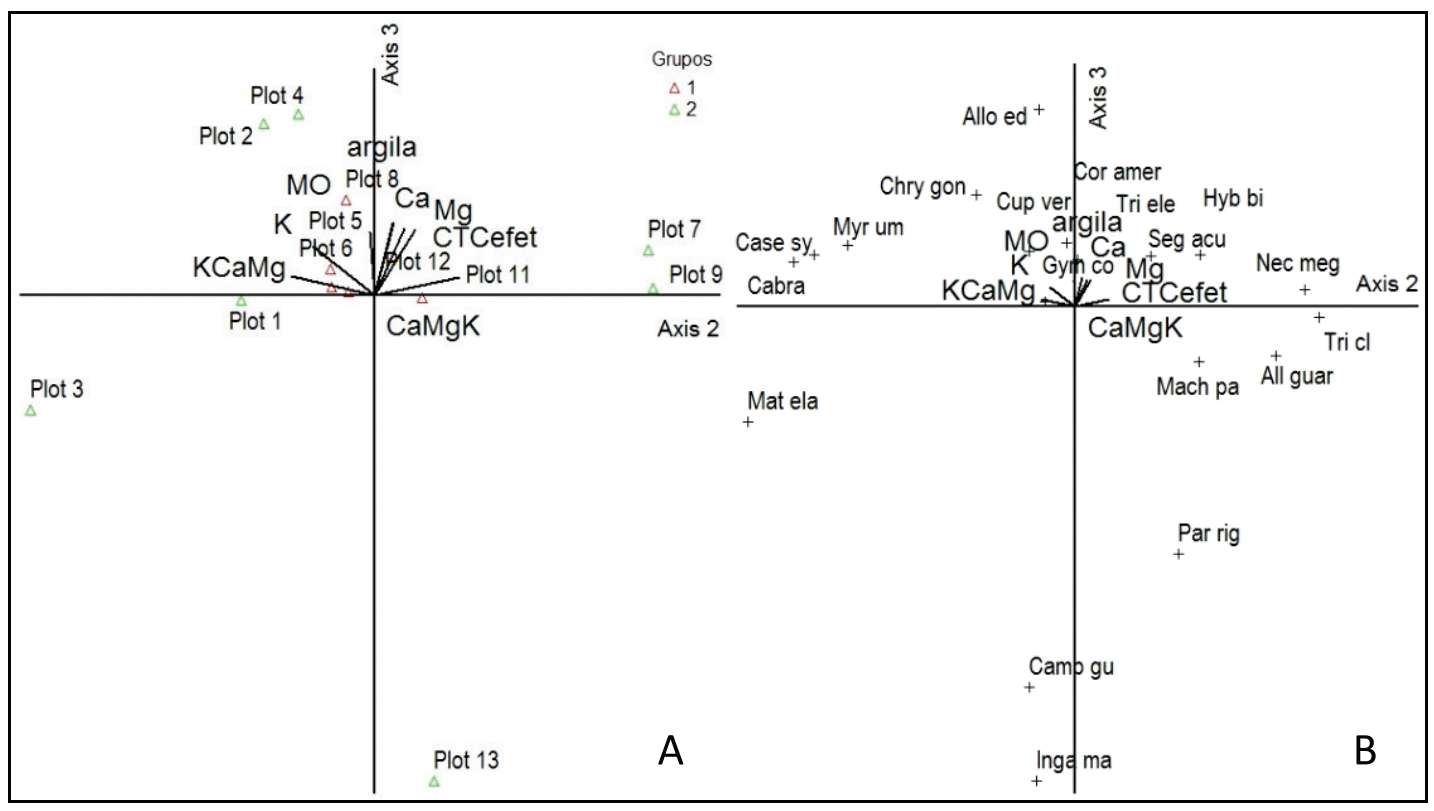

Figura 3 - Distribuição das parcelas (A) e espécies (B) de acordo com os eixos 3 e 2 da CCA, gerada a partir dos dados de abundância das espécies ocorrentes no sub-bosque e variáveis ambientais em um trecho de Floresta Estacional Subtropical no Parque Estadual Quarta Colônia, RS. Axis:eixos; Plot:parcelas; CTCefet: capacidade de troca catiônica efetiva; Ca: cálcio; PMehlic: fósforo; HAl:acidez potencial; Mg: magnésio; Tri ele:Trichilia elegans; Cab can:Cabralea canjerana; Allo ed:Allophylus edulis; All guar:Allophylus guaraniticus; Tri cla:Trichilia clausseni; Mach pa:Machaerium paraguaiensis; Cas syl:Casearia sylvestris; Cor ame:Cordia americana; Cam gua:Campomanesia guazumifolia; Nec meg:Nectandra megapotamica; Cup ver:Cupania vernalis; Myr umb:Myrsine umbellata; Mat elae:Matayba elaegnoides; Chry gon:Chrysophyllum gonocarpum; Para rig:Parapiptadenia rigida; Seg acu:Seguieria aculeata; Gym con:Actinostemon concolor; Inga mar:Inga marginata; Hyb bi:Hybanthus bigibbosus.

Figure 3 -Parcel distribution $(A)$ and species $(B)$ according to axes 3 and 2 of the CCa, generated from the data of abundance of species found in the understory and environmental variables in an excerpt from Semideciduous Subtropical in State Park Quarta Colônia, RS. CTCefet: effective cation exchange capacity Ca: calcium; PMehlic: phosphorus; HAl: potential acidity; Mg: magnesium Tri ele: Trichilia elegans; Cab can:Cabralea canjerana; Allo ed:Allophylus edulis; All guar:Allophylus guaraniticus; Tri cla:Trichilia clausseni; Mach pa:Machaerium paraguaiensis; Cas syl:Casearia sylvestris; Cor ame:Cordia americana; Cam gua:Campomanesia guazumifolia; Nec meg:Nectandra megapotamica; Cup ver:Cupania vernalis; Myr umb:Myrsine umbellata; Mat elae:Matayba elaegnoides; Chry gon:Chrysophyllum gonocarpum; Para rig:Parapiptadenia rigida; Seg acu:Seguieria aculeata; Gym con:Actinostemon concolor; Inga mar:Inga marginata; and Hyb bi:Hybanthus bigibbosus.

em Floresta Ombrófila Mista, encontraram menor diversidade no sub-bosque em relação ao componente arbóreo.

A formação dos grupos revela o grupo 1 do componente arbóreo com composição de espécies de sub-bosque, devido ao fato de o menor porte das árvores possibilitar seu estabelecimento em terreno declivoso e instável, características que se devem à intensidade de rochas fragmentadas. Provavelmente, a espécie indicadora desse grupo, Trichilia clausseni, exerce influência na sucessão da floresta e na comunidade em estudo, fato que pode ser explicado por diversos fatores ecológicos, como sua elevada densidade e frequência na regeneração da floresta (Tabela 1), grupo sucessional e interação planta-animal (STAGGEMEIER e GALETTI, 2007; MARTINS et al., 2007) e também por fatores ambientais, como luminosidade (REITZ et al., 1983), topografia e solo (SCIPIONI et al., 2010).

A composição de espécies indicadoras, como a Matayba elaeagnoides Radlk., e preferenciais, como Cupania vernalis, Ocotea puberula e Casearia sylvestris no grupo 2 do componente arbóreo evidencia maior

Revista Árvore, Viçosa-MG, v.37, n.2, p.275-287, 2013 
abundância de espécies secundárias iniciais no grupo, características de áreas em estágio intermediário de sucessão.

A espécie Matayba elaeagnoides Radlk. foi observada por Vaccaro et al. (1999) no estrato intermediário da floresta secundária, estando em constante competição, principalmente por luz. Conforme Brun et al. (2011), apresenta alto potencial de acumulação de carbono no solo, característica muito importante de espécies utilizadas em projetos de restauração e sequestro de carbono. Cupania vernalis teve representativa expressão, demonstrando que seu desempenho na área de estudo corrobora a afirmação de Lima et al. (2006), que a consideram espécie frequente em quase todas as formações florestais do bioma Mata Atlântica, devido à sua grande plasticidade em relação às diferentes intensidades luminosas, o que favorece seu estabelecimento em diferentes condições ambientais.

Quanto à estrutura da floresta nesse grupo, as espécies Nectandra lanceolata e Nectandra megapotamica foram dominantes na área. Cabe ressaltar que Nectandra megapotamica é uma das espécies que ocorrem na floresta densa e desenvolvida (REITZ et al., 1983) e apresenta regeneração vigorosa próxima de árvores adultas em senescência, de modo que sua presença indica que a área representada pelo grupo 2 se trata de um mosaico na floresta de sucessão avançada. As espécies Cupania vernalis, Ocotea puberula e Casearia sylvestris apresentam frutos atrativos para as aves (KRÜGEL et al., 2006), ocupando áreas de clareiras na floresta. Assim, para restauração de florestas, as referidas espécies apresentam importante função no ecossistema, já que são capazes de atrair a fauna e garantir sua ampla dispersão.

Os grupos formados no sub-bosque apresentaram a mesma classificação dos grupos do componente arbóreo, o que sugere que a floresta manterá a diferença dos grupos no decorrer da sucessão.

Considerando que as espécies presentes nesses grupos pertencem a diferentes grupos sucessionais, pode-se classificar o trecho estudado como uma floresta em estágio médio a avançado (CONAMA, 1994), onde as secundárias iniciais contribuem para maior diversidade e as espécies de sub-bosque possuem o maior número de indivíduos. As secundárias tardias aparecem em menor expressividade, tanto no número de espécies (18) quanto no número de indivíduos amostrados (150).
Dessa forma, essa classificação sugere a existência de um mosaico sucessional, em decorrência do histórico de interferência antrópica da área.

A propósito, a Floresta Estacional característica da região é decorrente das diferentes associações entre as espécies, locais de alta declividade, solos rasos, altas porcentagens de saturação de bases ( $>$ > 50\%) e baixos teores de alumínio (RUGGIERO et al., 2002), como verificado na área de estudo.

Diversos trabalhos sobre a importância de fatores ambientais na ocorrência das espécies demonstraram relações semelhantes àquelas aqui encontradas (BOTREL et al., 2002; MEURER, 2007; BUDKE et al., 2011). A abundância de espécies teve forte influência da variabilidade química e física do gradiente topográfico. Alguns autores observaram correlações da vegetação com a matéria orgânica (GIEHL; JARENKOW, 2008; ROSALES et al., 2001), em que esta possui a capacidade de adsorver $\mathrm{Ca}, \mathrm{Mg}$, $\mathrm{K}$ e outros elementos, evitando sua lixiviação em solos pobres de argila (KIEHL, 1979), como é o característico da área de estudo, que apresenta textura arenosa e altos teores de $\mathrm{Ca}, \mathrm{Mg}$ e $\mathrm{K}$.

Pedron e Dalmolin (2011) afirmaram que, no geral, os Neossolos Litólicos, característicos da área de estudo; e Regolíticos do rebordo do planalto gaúcho são eutróficos e ricos em matéria orgânica no horizonte A e em reserva de nutrientes, com exceção do fósforo.

Quanto ao sub-bosque, sua correlação com altos valores de potássio e relações molares indicam a necessidade de um aporte maior desses nutrientes, provavelmente devido à influência do sombreamento na produção de carboidratos para o desenvolvimento da planta (LARCHER, 2000).

A ordenação das unidades amostrais do componente arbóreo demonstrou que as parcelas situadas do lado esquerdo da ordenação correspondem ao grupo $1_{\mathrm{CA}}$ (Figura 2), onde provavelmente exista maior fertilidade do solo, devido à correlação com os altos teores de saturação de bases e CTC, pois o pH ligeiramente ácido e o elevado teor de $\mathrm{Ca}+\mathrm{Mg}$ trocável da área de estudo, conforme Malavolta (1997), favorecem a decomposição relativamente rápida da matéria orgânica, assegurando suprimento adequado de fósforo à vegetação mesmo que em baixas concentrações. Adicionalmente, as ordenações das espécies do componente arbóreo no grupo 1 apresentam as espécies Trichilia elegans,

Revista Árvore, Viçosa-MG, v.37, n.2, p.275-287, 2013 
Seguieria aculeata e Hybanthus bigibbosus correlacionadas com as variáveis de química de solo, as quais são comuns no sub-bosque e, de acordo com Lieberman et al. (1995), espécies do estrato herbáceoarbustivo estão aptas a ocupar a maior parte da amplitude de variação luminosa de seus ambientes, sofrendo, assim, maior influência por variáveis de solo do que por luminosidade na definição de nichos ecológicos das espécies de plantas nas florestas tropicais.

No eixo 3, as espécies Cordia americana, Cupania vernalis e Actinostemon concolor destacaram-se por sua correlação com potássio, matéria orgânica e relações molares de $\mathrm{K} /(\mathrm{Ca}+\mathrm{Mg})^{1 / 2}$. Nesse sentido, Scipioni et al. (2010) observaram que a espécie Cordia americana está ordenada em locais com maior relação $\mathrm{Ca} / \mathrm{Mg}$ e maior disponibilidade de cátions. Provavelmente, existe relação entre a deposição da serapilheira dessas espécies e a formação de matéria orgânica e disponibilidade de potássio (K), pois, conforme Malavolta (1997), o potássio apresenta alta mobilidade, sendo translocado facilmente da serapilheira para o solo e novamente para a planta. Cupania vernalis é espécie com alta deposição de serapilheira e concentração de macronutrientes (BRITEZ e SILVA, 1992).

Cada espécie tem intervalo de tolerância às variáveis ambientais, e quase sempre os limites dessa tolerância, ou seja, a disponibilidade de determinada variável, não são bruscos em um gradiente. Existe um ponto crítico a partir do qual a abundância das espécies vai diminuindo em relação aos extremos desse gradiente, que pode ser um recurso (luz, nutrientes) ou condições de hábitat (pH, altitude, topografia) (RODRIGUES et al., 2007). Com base nisso, pode-se explicar o fato de espécies que se distanciaram dos centros dos diagramas de ordenação terem menor exigência em relação às variáveis ambientais selecionadas.

Assim, diante dos agrupamentos encontrados e de suas espécies, como Cupania vernalis, Trichilia claussenii, Trichilia elegans, Allophylus edulis, Casearia sylvestris, Actinostemon concolor, Nectandra megapotamica, Nectandra lanceolata e Cordia americana, é possível identificar espécies potenciais à restauração. Essas espécies são importantes à restauração ecológica devido às suas características de plasticidade, distribuição espacial, frutificação atrativa para a fauna, ao grupo sucessional e à posição sociológica nos diferentes estratos, influenciando positivamente a intensidade luminosa e o estabelecimento de outras espécies na área a ser restaurada ou enriquecida. Entretanto, ressalta-se que a seleção da espécie ou a definição do arranjo depende da função que a espécie exerce no ecossistema e do resultado desejado.

\section{CONCLUSÃO}

a)A floresta no trecho estudado é heterogênea, e nela podem ser observados dois grupos florísticos, tanto no componente arbóreo quanto no sub-bosque. Os agrupamentos formados foram evidenciados, principalmente, pelo grupo sucessional ao qual pertencem as espécies predominantes, o que está relacionado ao histórico de uso do solo.

b) A distribuição da vegetação tem relação positiva com variáveis de solo, como cálcio, fósforo, potássio, magnésio, matéria orgânica, CTC, relações molares; e negativa, com acidez potencial e argila.

c) A relação de espécies com variáveis de solo diz respeito às características fisiológicas e ecológicas de cada espécie, como Cupania vernalis, Cordia americana e Actinostemon concolor, que se correlacionam positivamente com matéria orgânica e potássio, assim como Allophylus edulis, Banara tomentosa, Trichilia claussenii e Sebastiania brasiliensis com cálcio, fósforo e magnésio; Seguieria aculeata e Annona rugulosa se correlacionam negativamente com argila e acidez potencial.

d) Espécies como Cupania vernalis, Nectandra megapotamica, Casearia sylvestris, Trichilia clausseni e Cordia americana, que pertencem a diferentes grupos funcionais, são potenciais para serem utilizadas em programas de restauração, devido à sua capacidade de produzir serapilheira, ciclagem de nutrientes, sequestro de carbono, atrair fauna e dispersar sementes; assim como Actinostemon concolor e Trichilia elegans, que se apresentam como potenciais para compor a diversidade, por meio de enriquecimento.

\section{REFERÊNCIAS}

APG III. An update of the Angiosperm Phylogeny Group classification for the ordens and families of flowering plants: APG III. The Linnaean Society of London. Botanical Journal of the Linnean Socity, v.161, p.105-121, 2009. Disponível em: <http://onlinelibrary.wiley.com/doi/ 10.1046/j.1095-8339.2003.t01-1-00158.x/full>.

Acesso em: 4 out. 2011. 
BACKES, P.; IRGANG, B. Árvores do Sul: guia de identificação e interesse ecológico. Santa Cruz do Sul: Instituto Souza Cruz, 2002. 325p.

BOTREL, R. T. et al. Influência do solo e topografia sobre as variações da composição florística e estrutura da comunidade arbóreoarbustiva de uma floresta estacional semidecidual em Indaí, MG. Revista Brasileira de Botânica, v.25, n.2, p.195-213, 2002.

BRITEZ, R. M.; SILVA, S. M. Avaliação da regeneração natural em reflorestamentos experimentais da PETROSIX, São Mateus do Sul, PR. In: SIMPÓSIO NACIONAL SOBRE RECUPERAÇÃO DE ÁREAS DEGRADADAS, 7., 1992. Curitiba. Anais...Curitiba: UFPR/FUPEF, 1992. p.253-263.

BROWER, J. E.; ZAR, J. H. Field and laboratory methods for general ecology. 2.ed. Iowa: Brow Publishers, 1984. 226p.

BRUN, K. G. F.; BRUN, J. E.; LONGHI, L. S Potencial de sequestro de carbono em espécies arbóreas nativas em Santa Tereza. In: SCHUMACHER, M. V. et al. (Org.). A floresta estacional subtropical: caracterização e ecologia no rebordo do Planalto Meridional. Santa Maria: Pallotti, 2011.320p.

BUDKE, J. C.; JARENKOW, J. A.; OLIVEIRA FILHO, A. T. Relationships between tree component struture, topography and soil of a riverside Forest, Rio Botucaraí, Southern Brasil. Plant Ecology, v.189, n.2, p.187-200, 2007. Disponível em: <http:// www.springerlink.com/content/ e858717238387082/.>. Acesso em: 2 dez. 2011.

BUDOWSKI, G. N. Distribution of Tropical American rain forest species in the light of sucession processes. Turrialba, v.15, n.2, p.40-52, 1965.

CARVALHO, P. E. R. Espécies arbóreas brasileiras. Brasília: Embrapa Informações Tecnológicas; Colombo: Embrapa Floresta, 2003. 1039p. v.1.
CARVALHO, P. E. R. Espécies arbóreas brasileiras. Brasília: Embrapa Informações Tecnológicas; Colombo: Embrapa Floresta, 2006. 627 p. v.2.

CAUSTON, D. R. An introduction to vegetation analysis, principles and interpretation. London: Unwin Hyman, 1988. 340p.

CONNEL, J. H.; LOWMAN, M. D. Low-diversity tropical rain Forest: some possible mechanisms for their existence. The American Naturalist, v.131, n.1, p.88-119, 1989.

CONSELHO NACIONAL DO MEIO AMBIENTE CONAMA. Resolução n. 33 de 07 de dezembro de 1994. Diário Oficial [da] República Federativa do Brasil. n. 248, Poder Executivo, Brasília, DF, 30 dez. 1994. Seção 1. p.21352-53.

\section{EMPRESA BRASILEIRA DE PESQUISA} AGROPECUÁRIA - EMBRAPA. Centro Nacional de Pesquisa de Solos. Sistema brasileiro de classificação de solos. 2.ed. Rio de Janeiro: 2006. 306p.

FELFILI, J. M.; RESENDE, R. P. Conceitos e métodos em fitossociologia. Brasília: Universidade de Brasília, 2003. 68p.

FELFILI, J.M.; CARVALHO, F.A.; LIBANO, A.M.; VENTUROLI, F.; PEREIRA, B.A.S. Análise multivariada em estudos de vegetação. Comunicações Técnicas Florestais, v.9, n.1, Brasília, Departamento de Engenharia Florestal, Universidade de Brasília, 2007.

FELFILI, J. M. et al. Análise multivariada: princípios e métodos em estudos de vegetação. In: FELFILI, J. M. (Ed.). Fitossociologia no Brasil: métodos e estudos de casos. Viçosa, MG: Universidade Federal de Viçosa, 2011. v.1.

FINGER, C. A. Fundamentos de biometria florestal. Santa Maria: UFSM/CEPEF/FATEC, 1992. 269p.

Revista Árvore, Viçosa-MG, v.37, n.2, p.275-287, 2013 
GIEHL, E. L. H.; JARENKOW, A. J. Gradiente estrutural no componente arbóreo e relação com inundações em uma floresta ribeirinha, rio Uruguai, sul do Brasil. Acta Botânica Brasílica, v.22, n.3, p.741-753, 2008.

GRINGS, M.; BRACK, P. Árvores na vegetação nativa de Nova Petrópolis, Rio Grande do Sul. Iheringia, Série Botânica, v.64, n.1, p.5-22, 2009.

HILL, M. O. TWINSPAN: a FORTRAN program of arranging multivariate data in an ordered two way table by classification of individual and attibutes. Ithaca: Cornell University, 1979. 60p.

KIEHL, J. E. Manual de edafologia, relações solo-planta. São Paulo: Agronômica Ceres, 1979. 262p.

KILCA, V. R.; LONGHI, J. S. A composição florística e a estrutura das florestas secundárias no rebordo do Planalto Meridional. In: SCHUMACHER, M. V. et al. (Org.). A Floresta Estacional Subtropical: caracterização e ecologia no rebordo do Planalto Meridional. Santa Maria: Pallotti, 2011.320p.

KRÜGEL, M. M.; BURGER, M. I.; ALVES, M. A. Frugivoria por aves em Nectandra megapotamica (Lauraceae) em uma área de Floresta Estacional Decidual no Rio Grande do Sul, Brasil. Iheringia, Série Zoologia, v.96, n.1, p.17-24, 2006.

LARCHER, W. Ecofisiologia vegetal. São Carlos: Rima, 2000. 531p.

LIEBERMAN, M. et al. Canopy closure and the distribution of tropical forest tree species at La Selva, Costa Rica. Journal of Tropical Ecology, v.11, n.2, p.61-178, 1995. Disponível em: <http://hydro.csumb.edu/lieberman/docs/ Canopy\%20closure\%20and\%20the\%20distribution\%20JTE.pdfs. Acesso em: 4 set. 2011.

LIMA, E. C. et al. Aspectos fisioanatômicos de plantas jovens de Cupania vernalis Camb. Submetidos a diferentes níveis de sombreamento. Revista Árvore, v.30, n.1, p.33-31, 2006.
MALAVOLTA, E.; VITTI, G. C.; OLIVEIRA, S. A. Avaliação do estado nutricional das plantas. 2.ed. Piracicaba: Associação Brasileira para Pesquisa de Potássio e do Fosfato, 1997.

MARTINS, J. T.; WIDHOLZER, C. F. N.; DIAS, R. A. Comportamento da avifauna na captura e manipulação de frutos de Trichilia claussenii C. DC (Meliaceae) em mata de restinga do Rio Grande do Sul, BR. In: CONGRESSO DE INICIAÇÃO CIENTÍFICA, PESQUISA E RESPONSABILIDADE AMBIENTAL, 16., 2007, Pelotas. Anais... Pelotas: Faculdade de Agronomia Eliseu Maciel, 2007.

McCUNE, B.; MEFFORD, M. J. PC-ORD: multivariate analysis of ecological data. Version 5.0. Oregon: MjM Software Design, 2006.

MEURER, E. J. Fatores que influenciam o crescimento e o desenvolvimento das plantas. In: NOVAIS, R. F. et al. (Ed.). Fertilidade do solo. Viçosa, MG: Sociedade Brasileira de Ciência do Solo, 2007. p.65-90.

NARVAES, S. I.; BRENA, A. D.; LONGHI, J. S. Estrutura da regeneração natural em Floresta Ombrófila Mista na Floresta Nacional de São Francisco de Paula, RS. Ciência Florestal, v.15, n.4, p.331-342, 2005.

NIMER, E. Clima. In: INSTITUTO BRASILEIRO DE GEOGRAFIA E ESTATÍSTICA (IBGE).

Geografia do Brasil: Região Sul. Rio de Janeiro: 1990. p.151-187.

ORGANIZAÇÃO DAS NAÇÕES UNIDAS PARA AGRICULTURA E ALIMENTAÇÃO - FAO. Avaliação dos recursos florestais mundiais. 2010. Disponível em: <https:// www.fao.org.br/aif.asp>. Acesso em: 20 set. 2011.

PALMER, M. W. Putting things in even better order: the advantages of canonical correspondence analysis. Ecology, v.74, n.8, p.2215-2230, 1993.

PEDRON, A. P.; DALMOLIN, R.S. D. Solos da região do rebordo do Planalto Meridional no Rio Grande do Sul. In: SCHUMACHER, M. V. et al. (Org.). A Floresta Estacional Subtropical: caracterização e ecologia no rebordo do Planalto Meridional. Santa Maria: Pallotti, 2011.320p. 
REITZ, R.; KLEIN, R. M.; REIS, A. Projeto madeira do Rio Grande do Sul. Sellowia, v.34-35, p.5-483, 1983.

RODRIGUES, L. A. et al. Efeitos de solos e topografia sobre a distribuição de espécies arbóreaos em um fragmento de Floresta Estacional Semidecidual, em Luminárias, MG. Revista Árvore, v.31, n.1, p.25-35, 2007.

ROSALES, J.; PETTS, G.; KNAB-VISPO, C. Ecological gradients within the riparian forests of the lower Caura River, Venezuela. Plant Ecology, v.152, n.1, p.101-118, 2001.

RUGGIERO, P. G. C. et al. Soil-vegetation relationships in cerrado (Brazilian savanna) and semi-deciduous Forest, southeasterm Brazil. Plant Ecology, v.160, n.1, p.1-16, 2002. Disponível em: <http://

www.cerradoecology.com/papers_files/09.pdf $>$. Acesso em: 3 dez. 2011.

SCIPIONI, M. C. et al. Análise dos padrões florísticos e estruturais de uma comunidade arbórea-arbustiva em um gradiente de solo e relevo. In: SCHUMACHER, M. V. et al. (Org.). A Floresta Estacional Subtropical: caracterização e ecologia no rebordo do Planalto Meridional. Santa Maria: Pallotti, 2011. 320p.

SCIPIONI, M. C. et al.. Distribuição do compartimento arbóreo em gradiente de relevo e solos na encosta Meridional da Serra Geral, RS. Ciência Rural, v.40, n.6, p.1295-1301, 2010.
SCIPIONI, M. C. et al. Regeneração natural de um fragmento da Floresta Estacional Decidual na Reserva Biológica do IbicuíMirim (RS). Revista Floresta, v.39, n.3, p.675-690, 2009.

SECRETARIA ESTADUAL DO MEIO AMBIENTE SEMA/RS. Unidades de Conservação, Porto Alegre, 2011. Disponível em: <http://www.sema.rs.gov.br.

SILVA, A. L.; SCARIOT, A. Composição florística e estrutura da comunidade arbórea em Floresta Estacional Decidual em afloreamento de calcário (Fazenda S. José, São Domingos, GO, Bacia do Rio Paraná). Acta Botânica Brasileira, v.17, n.2, p.305-313, 2003.

STAGGEMEIER, V. G.; GALETTI, M. Impacto humano afeta negativamente a dispersão de sementes de frutos ornitocóricos: uma perspectiva global. Ararajuba. Revista Brasileira de Ornitologia, v.15, n.2, p.281-287, 2007.

TEDESCO, M. J. et al. Análises de solo, plantas e outros materiais.Porto Alegre: Universidade Federal do Rio Grande do Sul, 1995. 174p. (Série Boletim Técnico de Solos, v. 5).

VACCARO, J. S.; LONGHI, S. J.; BRENA, A. D. Aspectos da composição florística e categorias successionais do estrato arbóreo de três subseres de uma floresta estacional decidual, no município de Santa Tereza, RS. Ciência Florestal, v.9, n.1, p.1-18, 1999. 
